\title{
Philonsorbonne
}

12 | 2018

Année 2017-2018

\section{La simplicité monadique chez Leibniz}

Caroline ANGLERAUX

\section{OpenEdition}

Journals

Édition électronique

URL : https://journals.openedition.org/philonsorbonne/945

DOI : $10.4000 /$ philonsorbonne. 945

ISSN : 2270-7336

\section{Éditeur}

Publications de la Sorbonne

\section{Édition imprimée}

Date de publication : 1 janvier 2018

Pagination : $9-25$

ISSN : 1255-183X

\section{Référence électronique}

Caroline ANGLERAUX, «La simplicité monadique chez Leibniz », Philonsorbonne [En ligne], 12 | 2018,

mis en ligne le 03 mars 2018, consulté le 09 juin 2021. URL : http://journals.openedition.org/

philonsorbonne/945; DOI : https://doi.org/10.4000/philonsorbonne.945 


\title{
La simplicité monadique chez Leibniz
}

\author{
Caroline AnglerauX
}

\section{Introduction : les monades, unités substantielles}

Concept-clé de la philosophie leibnizienne de la maturité, la monade vient du grec monas signifiant «unité » et exprimant positivement ce que l'a-tomos expose par la négative du «ce qui ne peut être divisé ». Fidèle à cette étymologie, Leibniz caractérise ses monades en termes d'unités, comme dans une lettre à Rémond où il en fait des synonymes : «J'ai appris de M. Hugony, que vous trouvez quelque difficulté sur mes Unités ou Monades » 1 .

Les monades sont les unités de composition du monde naturel sans pour autant en être les composants ; elles informent le composé sans être ses parties. Aussi, les monades sont unités au sens de l'unité pleine du nombre entier, mais elles se distinguent de l'unité arithmétique, divisible en fractions et homogène au composé, en ce qu'elles sont insécables car hétérogènes aux composés qu'elles informent ${ }^{2}$. Elles sont les véritables atomes de la nature en ce qu'elles sont des unités indivisibles par-delà lesquelles on ne peut aller, mais elles diffèrent en tout de l'atome matériel ${ }^{3}$. Distinctes de l'unité

\footnotetext{
1. Toutes les citations de Leibniz sont issues de Die philosophischen Schriften von Leibniz, 7 vol., Berlin, C. I. Gerhardt, 1875-1890, noté par la suite GP suivi du numéro du volume. Ici, Leibniz à Rémond, GP III, p. 622.

2. Cf. M. Fichant, «La constitution du concept de monade », in La monadologie de Leibniz, Genèse et contexte, E. Pasini (éd.), Paris, Milan, Mimesis, 2005, p. 39.

3. Pour Leibniz, l'hypothèse d'un atome matériel est contradictoire. En effet, posant que la matière est divisible à l'infini, Leibniz avance qu'on ne peut fixer une entité matérielle indivisible qui, parfaitement identique à ses semblables, s'oppose de surcroît au principe de raison suffisante. Pour un exposé plus complet de la contradiction inhérente à l'atomisme
} 
arithmétique et de l'unité atomistique, les monades sont donc les véritables unités $^{4}$ de la nature, c'est-à-dire les unités qui fondent véritablement l'être des choses. En un mot, comme le résume Leibniz à Sophie, les monades sont unités de substance ${ }^{5}$.

Une fois le phénoménalisme et l'atomisme physique écartés par cette référence à la qualité substantielle des monades, l'existence de ces dernières tient, pour Leibniz, du réquisit rationnel, voire de l'impératif logique, que M. Fichant expose sous la forme d'un modus ponens dans ce qu'il appelle la «thèse monadologique " ${ }^{6}$ : il y a empiriquement des composés ; mais pour qu'il y ait des composés, que les composés soient, il faut ${ }^{7}$ des unités substantielles indivisibles; il y a donc des monades ${ }^{8}$. Les monades apparaissent ainsi comme les réquisits ${ }^{9}$ substantiels qui confèrent aux composés leur poids ontologique, et cet octroi substantiel se fait «par le bas » et «par le haut» :

Il n'y a que les Atomes de substance, c'est à dire, les unites reelles et absolument destituées de parties, qui soyent les sources des actions, et les [1] premiers principes absolus de la composition des choses, et comme les [2] derniers elemens de l'analyse des choses substantielles ${ }^{10}$.

physique pour Leibniz, cf. A.-L. Rey, «"Les Antipodes du pourquoi suffisant” ou comment rendre une fiction raisonnable? La dispute sur l'atomisme entre Leibniz et Hartsoeker », Les Études philosophiques, 163, (2016), p. 425-442.

4. Cf. « unités veritables et pure », GP VII, p. 552 ; «veritables Atomes de la Nature », GP VI, p. 607 ; « vrais Atomes de la nature », GP VI, p. 152.

5. «Cette Unité de substance ou cette Monade», Leibniz à Sophie, GP VII, p. 552. Cf. aussi «Unités de substance », GP VII, p. 565 ou « Atome de substance », GP IV, p. 478 et p. 482.

6. Cf. M. Fichant, « La constitution du concept de monade», op. cit., p. 32.

7. «Il faut nécessairement qu'il se trouve dans la nature corporelle des véritables unités », Leibniz, Systeme nouveau pour expliquer la nature des substances et leur communication entre elles, aussi bien que l'union de l'ame avec le corps, GP IV, p. 473, souligné par nos soins.

8. Cet argument est présenté relativement succinctement notamment dans le premier paragraphe des Principes de la Nature et de la Grace, fondés en raison, GP VI, p. 598 (« il faut bien qu'il y ait des substances simples par tout, parce que sans les simples il n'y auroit point de composés »), ou dans le second paragraphe de la Monadologie, GP VI, p. 606 (« Et il faut qu'il y ait des substances simples, puisqu'il y a des composés ; car le composé n'est autre chose, qu'un amas, ou agregatum des simples »). Il est parfois aussi développé plus amplement, comme dans la correspondance avec De Volder, GP II, p. 267 («Ad haec respondeo, primo argumentum meum repetendo distinctius hoc modo: Quae res in plura (actu jam existential) divide potest, ex pluribus est aggregate, et res quae ex pluribus aggregate est, non est unum nisi mente nec habet realitatem nisi a contentis mutuatam. Hinc jam inferebam, ergo dantur in rebus unitates indivisibiles, quia alioqui nulla erit in rebus unitas vera, nec realitas non mutuata. Quod est absurdum »).

9. «Monades [...] nec partes sunt corporum, sed requisite», Leibniz à Bierling, GP VII, p. 566, souligné par nos soins.

10. Leibniz, Systeme nouveau de la nature et de la communication des substances, aussi bien que l'union qu'il y a entre l'ame et le corps, GP IV, p. 482. Nous ajoutons [1] et [2]. 
Leibniz marque ici deux manières d'entendre l'unité monadique. [1] Dans un modèle intégratif, les monades sont considérées comme des unités qui confèrent une réelle unité per se aux composés, dans une attribution substantielle «par le haut». [2] Dans un modèle élémentaire, les monades sont appréhendées comme des unités de composition, dans une attribution substantielle «par le bas $»^{11}$. En ce sens, les monades sont pleinement unités substantielles, à la fois unités englobantes (du sens anglais de unity) et unités constituantes (du sens anglais de unit).

Ainsi les monades s'entendent-elles comme unités substantielles. Pourtant, alors que le terme de «monade » renvoie à la notion d'unité, Leibniz caractérise avant tout son concept central en termes de simplicité ; «La Monade, dont nous parlerons icy, n'est autre chose, qu'une substance simple» avance d'emblée Leibniz dans la Monadologie ${ }^{12}$. Mais tandis que l'unité qu'enferme la monade est distinguée de l'unité arithmétique, de l'atome physique ou encore du point mathématique, la simplicité qui caractérise la monade fait moins l'objet d'une distinction définitionnelle que celui d'une répétition ${ }^{13}$. En ce sens, pourquoi définir la monade comme simple ? Si la simplicité, et non l'unité, apparaît comme étant la qualité monadique discriminante, qu'apporte la simplicité à la monade alors même que l'appréhension de cette dernière en termes d'unité semble suffire à déterminer son contenu définitionnel ? L'objet de notre propos sera donc ici d'examiner le caractère simple de la monade leibnizienne pour en expliciter le contenu et comprendre sa place centrale dans la définition monadique.

\section{Simple, c'est-à-dire sans parties : la simplicité comme caractéristique fondamentale de l'unité véritable}

La définition, la plus succincte et la plus répétée dans les textes, que Leibniz attribue à la simplicité monadique est la qualité d'être sans parties : «La Monade, dont nous parlerons icy, n'est autre chose, qu'une substance simple $[\ldots]$; simple, c'est à dire, sans parties $»^{14}$, « la substance simple est

11. Sur la distinction de ces deux modèles, cf. M. Fichant, «L'invention métaphysique », in Discours de métaphysique suivi de Monadologie et autres textes, M. Fichant (éd.), Paris, Gallimard, 2004, p. 129 et P. Rateau, Leibniz et le meilleur des mondes possibles, Paris, Classiques Garnier, 2015, p. 187.

12. Leibniz, Monadologie, GP VI, p. 607.

13. Cf. pêle-mêle par exemple «aux monades, ou aux substances simples et à leurs modifications », GP VI, p. 590 ; «les Monades, ou substances simples », GP VII, p. 535 ; substantiae simplices seu monades, GP VII, p. 501; «Monas seu substantia simplex», GP VII, p. 502 ; «mes Monades ne sont pas des Atomes de matiere, mais des substances simples », GP III, p. 559: " des substances simples ou de véritables monades », GP VII, p. 356 ; «substances simples ou Monades», GP III, p. 662 : "Monada seu substantiam simplicem », GP II, p. 252 ; « l'assemblage des substances simples, ou des Monades », GP IV, p. 598 ; etc.

14. Leibniz, Monadologie, GP VI, p. 607. 
celle qui n'a point de parties $»^{15}$. Si la simplicité se réfère au fait d'être sans parties, alors la simplicité sort d'emblée le discours monadique de toute dialectique de la composition en termes de tout et de parties en soulignant l'hétérogénéité qui existe entre les monades et le composé qu'elles informent. En ce sens, si la simplicité s'appuie sur cette hétérogénéité, être simple implique immédiatement d'être indivisible. En d'autres termes, si la simplicité signifie «ce qui est sans parties », tandis que l'expérience montre que le composé peut être divisé en parties, alors la simplicité signifie «ce qui ne peut être divisé ». Cette implication de l'indivisibilité à la simplicité est ostensiblement accentuée par moments par l'adjonction de l'adjectif « indivisible » à celui de « simple »; « Il n'y a que les Monades, c'est à dire, les substances simples ou indivisibles ${ }^{16}$, «cela n'empêche point la matière d'être composée de substances simples et indivisibles $~^{17}$, «les substances véritablement simples et indivisibles ${ }^{18}$, par exemple. Conséquemment, si le simple est indivisible, il est immatériel par la même occasion. En effet, ce qui est sans parties est indivisible, mais ce qui est matériel est divisible à l'infini (selon la conception leibnizienne de la matière), donc ce qui est sans parties est immatériel. De même, si la simplicité implique l'immatérialité, elle enveloppe aussi l'impérissabilité ; puisque ce qui est matériel est périssable et puisque ce qui est simple est immatériel, alors ce qui est simple est impérissable ${ }^{19}$.

Ainsi, dire que les monades sont simples revient à avancer qu'elles sont à la fois indivisibles, immatérielles et impérissables ; une fois la simplicité posée, chaque qualité appelle la suivante, qui peut être déduite de la précédente. L'indivisibilité, l'immatérialité et l'impérissabilité constituent une sorte de triade qualitative ${ }^{20}$ qui se dévoile quand le contenu définitionnel de la simplicité est analytiquement déroulé.

Or indivisibilité, immatérialité et impérissabilité sont aussi les principales qualités de l'unité véritable que constitue la monade ; distincte de l'unité arithmétique, l'unité véritable est indivisible, et distincte de l'atome physique, elle est immatérielle. La simplicité comprend donc conceptuellement les attributs qui font de la monade une unité véritable.

15. Leibniz, Principes de la Nature et de la Grace, fondés en raison, GP VI, p. 598. Cf. aussi par exemple « une substance simple [...] laquelle n'ayant point de parties », GP VII, p. 657 ; «partibus carent», GP VII, p. 501 ; «partibus carent», GP VI, p. 529; «Cum omnem substantiam simplicem esse dico, hoc ita intelligo, ut partibus careat », GP II, p. 239.

16. Leibniz, Entretien de Philarete et d'Ariste, suite du premier entretien d'Ariste et de Theodore, GP VI, p. 585-586.

17. Leibniz à Sophie, GP VII, p. 561.

18. Leibniz, Théodicée, GP VI, p. 152.

19. «toute substance simple étant impérissable », Leibniz, Considérations sur les Principes de Vie, et sur les Natures Plastiques, par l'Auteur du Systeme de l'Harmonie preetablie, GP VI, p. 542.

20. Cf. encore «Et c'est cette substance simple, cette Unité de substance ou cette Monade, qu'on appelle Ame, et par conséquent les âmes, comme toute les autres Unités de substances, sont immatérielles, indivisibles et impérissables », Leibniz à Sophie, GP VII, p. 552-553. 
Mais selon le principe d'identité des indiscernables, deux choses ayant les mêmes attributs sont une seule et même chose ; simplicité et unité véritable ont les mêmes attributs, donc dire que la monade est simple équivaut à dire qu'elle est unité véritable. En cela, l'usage de la conjonction «ou» dans le Systeme nouveau de la nature et de la communication des substances, aussi bien que de l'union qu'il y a entre l'ame et le corps, témoigne de cette équivalence : «toute substance simple ou qui a une veritable unité ${ }^{21}$. De surcroît, comme nous l'avons posé en introduction, l'unité véritable est véritable en ce qu'elle fonde l'être des choses, en ce qu'elle est garante de leur réalité, de leur substantialité. En ce sens, en se présentant comme l'équivalent de l'unité véritable, la simplicité hérite de la réalité absolue inhérente à cette unité ${ }^{22}$ : être simple garantit donc d'être réel, c'est-à-dire d'être une substance.

Ainsi, définir succinctement la simplicité monadique comme ce qui est sans parties permet de déduire les principaux attributs monadiques : si ce qui est simple est sans parties, alors ce qui est simple est à la fois indivisible, immatériel et impérissable. Ce qui est simple est alors synonyme d'unité véritable. La simplicité monadique apparaît donc comme ce qui est garant de substantialité. En cela, comme rappelle P. Rateau, «la caractérisation de la substance par l'unité simple est, on l'a vu, le point d'aboutissement de la recherche d'un critère de la substantialité dans les corps tels qu'ils se donnent à voir dans l'expérience $\gg^{23}$; être simple garantit d'être substantiel.

Pour autant, ce premier examen de la simplicité semble mitigé car il permet tout au plus d'établir que la simplicité reprend les caractéristiques de l'unité véritable, sans ajouter davantage de contenu. En cela, la question se repose : qu'apporte de plus la simplicité que la caractérisation monadique comme unité véritable ne peut fournir? Au mieux peut-on dire pour l'instant que l'unité véritable n'est pas tant un synonyme de simplicité qu'un moyen terme pour déterminer la simplicité comme garant substantiel ; la simplicité est unité véritable, l'unité véritable est réalité absolue, et donc la simplicité est réalité absolue. L'unité véritable serait donc le lieu où simplicité et être se rencontrent ; comme l'avance A. Becco, «c'est autour de SIMPLE qu'il convient finalement de dire que ens et unum convertuntur $»^{24}$. En ce sens, on peut noter que dans l'Entretien de Philarete et d'Ariste, Leibniz élude l'unité véritable pour qualifier directement l'âme (et donc toute

21. Leibniz, Systeme nouveau de la nature et de la communication des substances, aussi bien que de l'union qu'il y a entre l'ame et le corps, GP IV, p. 479. Cf. aussi « une substance simple, ou Unité », Leibniz à Sophie, GP VII, p. 567 ou encore « une multitude dans l'unité ou dans la substance simple », Leibniz, Monadologie, GP VI, p. 608.

22. Leibniz à De Volder, GP II, p. 275.

23. P. Rateau, Leibniz et le meilleur des mondes possibles, op. cit., p. 190.

24. A. Becco, Du simple selon G. W. Leibniz: Discours de Métaphysique et Monadologie : étude comparative critique des propriétés de la substance, appuyée sur l'opération information "Monado 74”, Paris, Vrin, 1975, p. 116. 
substance simple) de « réalité immatérielle, indivisible et indestructible ${ }^{25}$, ce qui pourrait inciter à voir l'unité véritable comme un moyen terme plus qu'un synonyme. De même, quand Leibniz écrit à De Volder que seules les substances simples ont une unité et une réalité absolue (substantiis simplicibus quae solae unitatem et absolutam realitatem habent $)^{26}$, on peut noter que les substances simples sont seules à avoir une unité et une réalité absolue, faisant ainsi des substances simples l'élément auquel «unité »se rapporte au même titre que "réalité absolue », et donc sans mettre la notion d'unité véritable sur le même plan que celle de simplicité. Pour autant, cette précision semble encore mince pour comprendre que la simplicité englobe davantage l'unité qu'elle en est le strict synonyme. En cela, comment entendre pleinement la primauté du simple sur l'unité véritable?

\section{La nature du simple est d'avoir de la perception : la simplicité riche de complexité}

Dans une lettre adressée à Sophie, Leibniz propose une autre définition de la simplicité monadique, tout aussi lapidaire que celle par l'absence de $\operatorname{parties}^{27}:$ «V.A.E. me demande ce que c'est qu'une Substance simple. Je réponds que sa nature est d'avoir de la perception et par consequent de representer les choses composées $»^{28}$. Ici être simple, c'est avoir de la perception, c'est-à-dire être capable d'appréhender la multitude dans une unité $^{29}$. En d'autres termes, être simple c'est avoir de la perception, c'est-àdire être capable de faire d'une diversité une série compréhensible. En effet, comme l'explique A. M. Nunziante, la perception transforme des données collectées dans le divers de l'environnement en une seule unité représentative; en cela, l'acte de percevoir met en place un système de relations qui permet d'ajouter chaque donnée entrante, aussi minime soit-

25. Leibniz, Entretien de Philarete et d'Ariste, suite du premier entretien d'Ariste et de Théodore, GP VI, p. 588.

26. Leibniz à De Volder, GP II, p. 275.

27. Ce deuxième volet définitionnel, quoique proposant un nouveau parcours interprétatif que nous suggérons d'emprunter, reste solidaire de l'appréhension de la simplicité en termes d'absence de parties, la simplicité informant les composés dans les deux cas.

28. Leibniz à Sophie, GP VII, p. 566. Ici, nous ouvrons ce moment avec cet extrait au contenu explicite, mais l'appréhension de la simplicité comme ce qui a de la perception apparaît ailleurs dans le corpus leibnizien, comme par exemple : "nihil in rebus esse nisi substantias simplices et in his perceptionem atque appetitum », GP II, p. 270 ; «naturellement chaque substance simple a de la perception», GP VI, p. 289 ; «Porro Monas seu substantia simplices in genere continet perceptionem et appetitum », GP VII, p. 502.

29. En effet, la perception est définie par Leibniz, notamment dans sa correspondance avec Rémond (GP III, p. 622), avec Bourguet (GP III, p. 574) ou dans la Monadologie (GP VI, p. 608) comme « la représentation de la multitude dans le simple». 
elle, en l'ordonnant dans une structure de différences et de connections ${ }^{30}$. Ainsi, cette manière d'ordonner les données entrantes dans un canevas agençant différences et similarités construit un tissu perceptif propre à chaque substance simple, tissu perceptif qui fonde la singularité de chaque substance simple considérée. Loin de présenter une totalité permanente et immobile, ce tissu perceptif tient du flux car, naturellement, «toute perception présente tend à une perception nouvelle ${ }^{31}$; ce passage spontané d'une perception à l'autre se fait «par les loix des Appetits » ${ }^{32}$, c'est-à-dire par une inclination, une tendance active, un impetus ${ }^{33}$ qui fait spontanément passer d'une perception à l'autre au sein du tissu perceptif fluide.

En ce sens, définir la simplicité monadique par le fait d'avoir de la perception met en lumière la fluidité du contenu monadique et évite toute lecture qui inciterait à voir une fixité immobile au sein des substances simples qui, par la même occasion, seraient simples dans leur constitution. En effet, si les simples monadiques sont simples par constitution, alors rien ne change et le monde resterait parfaitement immobile ; comme l'écrit Leibniz à De Volder, «il n'y aurait pas de changement du tout dans les choses, s'il n'y en avait pas dans les simples $»^{34}$. Or si l'expérience nous fait constater que les composés changent, et si l'on pose que les substances simples sont les réquisits des composés, alors les substances simples doivent changer. En cela, les simples doivent contenir une puissance de changement, à savoir, la puissance de percevoir et, corrélativement, d'appéter ${ }^{35}$.

En ce sens, appréhender ce qui est simple comme ce qui a de la perception montre que la simplicité monadique dit plus que l'unité véritable. En ce que le simple est ce qui est sans parties mais aussi ce qui a de la perception, la simplicité met à la fois l'accent sur les qualités monadiques (indivisible, immatérielle, impérissable, unité véritable, garante de substantialité) et sur la puissance de changement induite par la perception et

30. «The perception, indeed, turns the manifold environmental inputs into a single representative unit [...] the perceiving act as the setting up of a system of relations, which permits the acquisition of every minimal environmental input by ordering it into a framework of differences and connections », A. M. Nunziante, "The Concept of "Organism" in EarlyModern Western Philosophy. Leibniz, the Cartesian Machines and the Aristotelian tradition », présenté le 21 août 2015 à la Hokkaido University.

31. Leibniz, Théodicée, GP VI, p. 356-357.

32. Leibniz, Principes de la Nature et de la Grace, fondés en raison, GP VI, p. 598.

33. «Each appetition is a tendency, desire or urge that moves the monad from one perception to the next », P. Phemister, Leibniz and the Natural World, Netherlands, Springer, 2005, p. 138.

34. patet nisi in simplicibus mutation sit, mutationem in rebus omnio nullam fore, Leibniz à De Voler, GP II, p. 252. Traduction A.-L. Rey, Leibniz-De Volder Correspondance, Paris, Vrin, 2016, p. 229.

35. «Sans parties, le simple comporte cependant un principe de changement interne, de la perception et de l'appétition », A. Becco, Du simple selon G. W. Leibniz: Discours de Métaphysique et Monadologie : étude comparative critique des propriétés de la substance, appuyée sur l'opération information "Monado 74", op. cit., p. 96. 
l'appétition, c'est-à-dire sur l'activité monadique interne foisonnante ${ }^{36}$ où certains détails changent et d'autres persistent. La simplicité n'est donc pas tant synonyme de l'unité véritable qu'elle n'en fait une composante de sa notion.

D'autant plus que considérer que ce qui est simple est ce qui a de la perception aboutit, tout comme la qualification du simple par l'absence de parties, à conclure qu'être simple garantit d'être substantiel. En effet, nous l'avons vu, appréhender la simplicité par le fait d'avoir de la perception caractérise d'emblée ce qui est simple par ce qui est actif, le flux perceptif étant garant d'une foisonnante activité monadique. Or pour Leibniz, «la Substance est un Etre capable d'action $»^{37}$, ou encore, « ce qui n'agit, ce qui est dépourvu de puissance active, de toute marque distinctive, en un mot ce qui est privé de toute raison de subsister, cela ne peut en aucune façon être une substance $»^{38}$; ce qui est authentiquement actif est substantiel. En entendant ce qui est simple comme ce qui a de la perception, la simplicité apparaît donc une fois encore comme le noyau substantiel et le garant de la substantialité. De manière peut être plus évidente encore, quand Leibniz avance que les monades sont faites d'une puissance primitive active et d'une puissance primitive passive et alors que la puissance primitive passive tient un simple rôle limitatif ${ }^{39}$, la puissance primitive active apparaîtrait comme le noyau actif de la substance simple. En témoigne par exemple une lettre à Lady Masham dans laquelle Leibniz rend directement synonyme la substance simple et la force primitive : «l'idée positive de cette substance simple ou Force primitive est toute trouvée ${ }^{40}$; ici, rendre synonymes les deux termes souligne le rôle substantiel clé de la force primitive ${ }^{41}$, lieu d'activité authentique résultant du tissu perceptif, et donc du simple. En ce sens, que le simple soit entendu comme ce qui est sans parties ou ce qui

36. Cf. « Si admittamus unam substantiam in aliam influere non posse, quod multi concedunt, jam hinc sequitur substantiam quamvis esse per se activam », Leibniz à De Volder, GP II, p. 206 et aussi « Aussi n'y a-t-il que cela qu'on puisse trouver dans la substance simple, c'est à dire les perceptions et leur changemens. C'est en cela seul aussi que peuvent consister toutes les Actions internes des substances simples », Leibniz, Monadologie, GP VI, p. 609.

37. Leibniz, Principes de la Nature et de la Grace, fondés en raison, GP VI, p. 598.

38. «Id quod non agit, quod vi activa caret, quod disciminabilitate, quod denique omni subsistendi ratione ac fundamento spoliatur, substantia esse nullo modo possit », Leibniz, De Ipsa Natura, GP IV, p. 515. Traduction P. Schrecker, Leibniz, Opuscules philosophiques choisis, Paris, Vrin, 2001, p. 233.

39. «Creatures must possess this limiting passive force if they are to be distinguished both from their Creator and from each other. A creature whose perceptual states were identical to God's would, since beings are distinguished according to their perceptions and appetitions, be indistinguishable from God and, by the Principle of the Identity of Indiscernibles, would be one and the same with God [...] then, without primitive passive force or primary matter to restrain the individual creature's active force, there would be no variation in the world», P. Phemister, Leibniz and the Natural World, op. cit., p. 242.

40. Leibniz à Lady Masham, GP III, p. 356.

41. «The primitive force itself is the principle or law that encapsulates the susbtance's essence », P. Phemister, Leibniz and the Natural World», op. cit., p. 134. 
a de la perception, dans les deux cas, le simple apparaît comme le noyau substantiel et le garant de la substantialité.

En outre, de manière générale, définir la simplicité par le fait d'avoir de la perception insiste sur la richesse du contenu monadique ${ }^{42}$. Comme Leibniz l'énonce, « la simplicité de la substance n'empeche point la multiplicité des modifications ${ }^{43}$, c'est-à-dire la multiplicité des changements perceptifs internes. Cette richesse du contenu de la substance simple est interne au simple, défini par l'activité perceptive qu'il renferme. En cela, la simplicité monadique n'est pas constitutivement simple mais riche de complexité. Posons de nouveau les choses. Les substances simples, informant les composés, doivent nécessairement contenir une puissance de changement pour que le changement empiriquement constaté dans les composés soit explicable. Cette puissance de changement prend sens dans l'activité perceptive qui est contenue dans le simple, car le simple est ce qui a de la perception. Caractérisé ainsi par le fait d'avoir de la perception, le simple monadique est donc fluide, informatif, créateur de changement. Le simple monadique est donc complexe par sa constitution. C'est même à condition d'être complexe que la monade est un simple créateur, actif et non inerte.

Ici, il nous semble plus adéquat de parler de complexité dans la simplicité que de composition ou de multitude dans la simplicité. En effet, la composition véhicule étymologiquement l'idée d'un assemblage partes extra partes et donc insiste sur une composition du composé en plusieurs parties. De même, la multiplicité se concentre sur l'idée du «beaucoup », du grand nombre. Dans les deux cas, l'unité, la cohésion monadique, semble pâtir. La notion de complexité, quant à elle, suppose une constitution multiple, faite de plusieurs liens différents. Mais de surcroît, le complexe est cum-plexus : les nombreux fils s'y entrelacent, s'y tissent pour ne former qu'une seule entité ; l'unité, la cohésion de la substance simple est donc ici préservée. En outre, on peut noter que le complexe autorise une certaine diversité dans les éléments qu'il tisse, soulignant peut-être par-là même la diversité des perceptions dont les détails changent et perdurent ${ }^{44}$.

Aussi, appréhender la simplicité monadique comme riche de complexité réaffirme d'autant plus sa simplicité. Du latin simplex qui conjugue l'un, le même du sim-/sem- (semel) et le tissage d'une pluralité que suppose le -plex

42. «La perception remplit cette fonction remarquable d'assurer la fécondité du simple», A. Becco, Du simple selon G. W. Leibniz: Discours de Métaphysique et Monadologie : étude comparative critique des propriétés de la substance, appuyée sur l'opération information "Monado 74", op. cit., p. 152.

43. Leibniz, Principes de la Nature et de la Grace, fondés en raison, GP VI, p. 598.

44. «Cette simplicité compositionnelle n'exclut pas toutefois une forme de complexité interne, sous la forme d'une diversité qualitative. Cette diversité est à la fois synchronique, issue de la représentation par chaque monade de l'ensemble des autres monades, et diachronique, en ce que chaque monade est sujette au changement», J.-P. Anfray, «Le labyrinthe temporel, Simplicité, persistance et création continuée chez Leibniz », Archives de Philosophie, 77, (2014), p. 43. 
venant de plico et désignant l'action d'enrouler, de plier, le simple suppose étymologiquement une complexité originelle. En ce sens, la simplicité monadique suit à la lettre son étymologie; forte du passé et grosse de l'avenir ${ }^{45}$, la substance simple contient enroulées en elle toutes ses perceptions que le temps a pour rôle d'exprimer, de dérouler.

Par ses perceptions, des plus distinctes aux plus confuses, par ses repris monadiques allant à l'infini, la substance simple est un miroir vivant de l'univers qu'elle exprime sous son point de vue propre. En ce sens, Leibniz avance : "chaque substance simple est un miroir du même Univers, aussi durable et aussi ample que luy, quoyque ces perceptions des Creatures ne sauroient être distinctes qu'à l'egard de peu de chose à la fois et qu'elles sont diversifiées par les rapports ou pour ainsi dire, par le point de veue des miroirs, ce qui fait qu'un même Univers est multiplié d'une infinité de façons par autant de miroirs vivans, chaque se representant à sa mode ${ }^{46}$. Dans cette veine s'engage une métaphore du miroir très élaborée; chaque substance simple constitue un tissu perceptif singulier, solidaire de ceux des autres substances simples, et formant tous ensemble les fibres du tissu de l'univers ${ }^{47}$. Reflet reflété, la pensée du simple complexe outrepasse plusieurs cadres de pensée : les simples forment les complexes et les complexes sont dans les simples, la singularité fait le pluriel et le pluriel est contenu dans les singularités, chaque monade fait le tout du monde et le tout du monde est dans chaque monade; l'intériorité monadique pose l'extériorité et l'extériorité est condition de l'intériorité monadique ${ }^{48}$. En ce que le simple est ce qui a de la perception, la simplicité monadique se dévoile dans toute sa com-plexitét ${ }^{49}$; pour paraphraser M. Fichant, dans la détermination ontologique de la substance par la simplicité, nous atteignons la simplicité de la chose et non de sa notion ${ }^{50}$.

45. «Et comme tout présent état d'une substance simple est naturellement une suite de son état précédent, tellement que le présent y est gros de l'avenir », Leibniz, Monadologie, GP VI, p. 247.

46. Leibniz à Rémond, GP III, p. 623.

47. «L'activité perceptive est eo ipso relation mondiale par excellence, la loi de l'existant en général. Le monde est la réunion ou l'intersection des présentations monadiques », M. Serres, Le Système de Leibniz et ses modèles mathématiques, tome II, Paris, PUF, 2007, p. 147.

48. «L'intériorité monadique n'est pas à penser par opposition à une extériorité (les autres monades ou plus généralement le "monde extérieur") ; elle n'a d'intériorité que parce que l'extériorité est en elle », J. Roland, Leibniz et l'individualité organique, Montréal, Paris, Presses de l'Université de Montréal, Vrin, 2012, p. 273.

49. Leibniz's sense of simplicity denies complexity full-stop, T. A. Hillman, « Substantial simplicity in Leibniz: form, predication, \& truthmakers », The Review of Metaphysics, 63, (2009), p. 92.

50. M. Fichant, «L'invention métaphysique », op. cit., p. 136. 


\section{Substances simples et substances composées : la simplicité comme noyau ontologique}

Ainsi et pour retracer le chemin parcouru, en ce que le simple est ce qui est sans parties, la simplicité suppose à la fois l'indivisibilité, l'immatérialité et l'indestructibilité ; en cela, la simplicité récapitule les attributs de l'unité véritable et hérite du rôle de cette dernière : la simplicité est garante de substantialité, est simple ce qui est substantiellement. Corrélativement, en ce que le simple est ce qui a de la perception, c'est-à-dire ce qui est authentiquement actif, et en ce que «toute substance est active ${ }^{51}$, la simplicité apparaît encore une fois comme le noyau de la substantialité monadique. En ce sens, le simple se présente comme l'attribut central monadique et la désignation des monades en tant que substances simples prend pleinement sens. Pour autant, si de la simplicité découle nécessairement la substantialité, il ne s'ensuit pas que la substantialité se cantonne au simple. Alors que, dans la Monadologie, la monade est directement présentée comme un simple - et rend par là-même l'expression de «substance simple» redondante, voire pléonastique ${ }^{52}$-, dans les Principes de la Nature et de la Grace et dans d'autres textes ${ }^{53}$, Leibniz aborde la question des substances composées, constituées de substances simples et de corps organiques ${ }^{54}$. Or, pour Leibniz, Dieu mis à part, à toute substance simple est joint un corps organique: « une entéléchie primitive ne peut jamais naître naturellement ou s'éteindre, elle n'est, en outre, jamais privée de corps organique $»^{55}$, ou encore «chaque Ame ou Monade est toujours accompagnée d'un corps organique $»^{56}$. En ce sens, si aux substances simples créées sont adjoints des corps organiques et si l'ensemble forme des substances composées, la simplicité peut-elle encore constituer le garant de la substantialité, le noyau substantiel ? En ce sens, ce dernier volet de notre enquête vise à mettre en lumière la conséquence de la définition du simple comme ce qui a de la perception, à savoir représenter les choses composées.

En effet, rappelons-le: «V.A.E. me demande ce que c'est qu'une Substance simple. Je réponds que sa nature est d'avoir de la perception et par consequent de representer les choses composées ${ }^{57}$. En d'autres termes, si le

51. Leibniz à Rémond, GP III, p. 657.

52. Leibniz, Monadologie, GP VI, p. 607. Cf. aussi par exemple l'indétermination de la re simplici dans une lettre à Wagner, GP VII, p. 529.

53. Cf. par exemple GP II, p. 260 ; GP IV, p. 572 ; GP VI, p. 550 ; GP VII, p. 501.

54. «Substantiae compositae sunt quae unum per se constituent ex anima et corpore organico », Leibniz à Des Bosses, GP II, p. 439.

55. «Nec unquam oriri naturaliter aut extingui potest primitive Entelechia quaecumue, et nunquam organico corpore caret », Leibniz à De Volder, GP II, p. 251. Trad. A.-L. Rey, Leibniz-De Volder Correspondance, op. cit., p. 227.

56. Leibniz à Des Maizeaux, GP VII, p. 535.

57. Leibniz à Sophie, GP VII, p. 566. 
simple est ce qui a de la perception, et si la perception est l'appréhension du multiple dans l'unité, alors avoir de la perception permet de représenter l'ensemble de l'univers sous son point de vue propre ; «chaque Monade creée represente tout l'univers $»^{58}$. En cela, si le simple est ce qui a de la perception, et si avoir de la perception permet de représenter les choses composées, alors le simple permet de représenter les choses composées. Mais, pour que chaque substance simple puisse représenter sous son propre point de vue les choses composées, son activité perceptive doit trouver un support expressif, à savoir le corps organique qui lui est attribué. Ainsi, le corps organique assigné à une monade déterminée lui donne les moyens de la réalisation de son expression monadique ; le corps organique constitue un intermédiaire par lequel la substance simple peut concrètement ${ }^{59}$ réaliser son activité perceptive ${ }^{60}$; comme Leibniz l'avance, « et en outre chaque monade créée est pourvue d'un corps en un sens organique, et selon lequel elle perçoit et elle appète ${ }^{61}$. En cela, la substantialité monadique ne dépend pas du corps organique mais, en tant qu'entité métaphysique inétendue et donc physiquement non localisée, la substance simple voit sa réalisation effective passer par le corps organique ${ }^{62}$.

Et cette réalisation effective est rendue possible par le fait que la constitution du corps organique est adéquate au contenu perceptif à exprimer $^{63}$. En ce sens, de même que les replis monadiques vont à l'infini, de même la composition organique va à l'infini ; «les Machines de la Nature, c'est à dire les corps vivans, sont encor des machines dans leur moindres

58. Leibniz, Monadologie, GP VI, p. 617.

59. «La réalité monadique ne peut prendre consistance que dans l'existence de composés que sont les vivants », J. Roland, Leibniz et l'individualité organique, op. cit., p. 324 (souligné par nos soins).

60. «Il faut lier l'animation à l'organicité des corps, donc à une structure auto-suffisante pour la production d'effets correspondant au dynamisme interne des monades », F. Duchesneau, Leibniz, le vivant et l'organisme, Paris, Vrin, 2010, p. 185. En effet, l'expression de cette puissance active primitive qu'est l'activité perceptive par l'intermédiaire des forces dérivatives est réalisée par la structure organisée du corps organique; le corps organique permet de rendre effective l'activité monadique par les forces dérivatives qui, par la même occasion, témoignent de la présence de l'activité monadique - «a body's derivative force, therefore serves to indicate the monadic primitive active forces that have come together for (concurrent ad) that moving body» (P. Phemister, «Monads and Machines », in Machines of Nature and Corporeal Substances in Leibniz, J. E. H. Smith, O. Nachtomy (éd.), Netherlands, Springer, 2011, p. 47-48).

61. «Et praeterea omnis Monas creata est corpore aliquo organico praedita, secundum quod percipit appetitque », Leibniz à Bierling, GP VII, p. 502. Traduction par nos soins.

62. «We cannot understand monads as entelechies or soul-like unifying forces when there are no further organisms in which the forces can be exhibited», H. Ishiguro, «Unity without Simplicity : Leibniz on Organisms », The Monist, 81, (1998), p. 546.

63. «Pour que chaque monade exprime l'univers infini tout entier sous le point de vue que définit son corps, il faut que ce corps exprime aussi dans sa structure la richesse et la complication de l'univers », M. Fichant, «Leibniz et les machines de la nature », Studia Leibnitiana, 35, (2003), p. 14. 
parties jusqu'à l'infini $»^{64}$. Cette composition à l'infini fait du corps organique une structure hautement adaptative ; le corps persiste par-delà les changements, tout comme les monades restent les mêmes au fil des changements que sa constitution perceptive suppose ${ }^{65}$. En outre, cette composition à l'infini implique ${ }^{66}$ que le corps organique est aussi impérissable, comme c'est le cas pour les monades ${ }^{67}$. Par sa composition, le corps organique se présente donc comme un support expressif adéquat et proportionnel à l'activité perceptive propre à chaque substance simple «ces Ames ou Entelechies ont toutes une manière de corps organique avec elles proportionné à leur perceptions ${ }^{68}$.

Ainsi, avoir de la perception revient à avoir la capacité de conjuguer la multiplicité dans l'unité et, en cela, à représenter les choses composées. Pour que cette représentation puisse concrètement se réaliser, l'activité perceptive doit donc s'incarner par un support expressif adapté, structurellement compatible à l'expression du contenu monadique, à savoir un corps organique. En ce sens, si être simple est avoir de la perception, et donc par conséquent représenter les choses composées, alors le simple doit s'accompagner d'un corps organique et, de ce fait, constituer une substance composée ${ }^{69}$.

Et cette substance composée est donc faite d'une imbrication de substances simples et de corps organiques. En effet, en considérant la

64. Leibniz, Monadologie, GP VI, p. 618.

65. «Une machine naturelle demeure encor machine dans ses moindres parties, et qui plus est, elle demeure tousjours cette même machine qu'elle a esté, n'estant que transformée par des differens plis qu'elle reçoit, et tantost étendue, tantost resserrée et comme concentrée lorsqu'on croit qu'elle est perdue », Leibniz, Systeme nouveau de la nature et de la communication des susbtances, aussi bien que l'union qu'il y a entre l'ame et le corps, GP IV, p. 482.

66. «Leibniz makes it very clear that the indestructibility of natural machines derives from their composition to infinit»y, O. Nachtomy, «Leibniz on Artificial and Natural Machines : On What It Means to Remain a Machine to the Least of Its Parts », in Machines of Nature and Corporeal Substances in Leibniz, J. E. H. Smith, O. Nachtomy (éd.), op. cit., p. 76.

67. «Je tiens non seulement que ces Ames ou Entelechies ont toutes une manière de corps organique avec elles proportionné à leur perceptions, mais même qu'ils en auront tousjours et en ont tousjours eu, tant qu'elles ont existé [...]. Et par consequent ny le fer ny le feu, ny toutes les autres violences de la nature, quelque ravage qu'elles fassent dans le corps d'un animal, ne sauroient empecher l'ame de garder un certain corps organique », Leibniz à Lady Masham, GP III, p. 340.

68. Leibniz à Lady Masham, GP III, p. 340. Et ce rapport proportionnel est garanti par l'ordre organique. En effet l'organique, l'organisme est avant tout synonyme d'ordre - « l'Organisme c'est à dire l'ordre et l'artifice », Leibniz à Lady Masham, GP III, p. 340 - et réfère à la fois (1) à l'organisation à l'infini de la matière organique corporelle et (2) à l'organisation de leur rapport. En ce sens, il y a une harmonie expressive générale entre les substances simples et les corps organiques selon laquelle "ratumque est quod in anima, idem in corpore exprimi» (Leibniz à De Volder, GP II, p. 251.

69. «His [Leibniz's] doctrine of simple substances requires the existence of corporeal substances », S. Levey, «On Unity and Simple Substance in Leibniz », The Leibniz Review, 17, (2007), p. 63. 
structure infinie de l'organisme, chaque partie d'un corps organique est ellemême structurée à l'infini ; chaque corps organique est donc lui-même composé d'une infinité de corps organiques, chaque corps étant lui-même structuré par d'autres corps organiques, et ce à l'infini. Or, chaque corps organique est joint à une substance simple. Donc, comme Leibniz le pose : « il est vray que le nombre des substances simples qui entrent dans une masse quelque petite qu'elle soit, est infini, puisqu'outre l'ame qui fait l'unité reelle de l'animal, le corps du mouton (par exemple) est soubsdivisé actuellement, c'est à dire qu'il est encor un assemblage d'animaux ou de plantes invisibles, composés de même outre ce qui fait aussi leur unité reelle ${ }^{70}$. Ainsi, une substance composée est constituée d'une imbrication de substances simples et de corps organiques à l'infini. Conséquemment, cette imbrication à l'infini fait des substances simples et des corps organiques de mêmes entités, à savoir des substances composées. De ce fait, il n'y a pas de dualisme chez Leibniz, qui distinguerait d'un côté les substances simples et de l'autre les corps organiques ; substances simples et corps organiques constituent ensemble, imbriqués, les êtres vivants ${ }^{71}$. C'est peut-être en ce sens que Leibniz écrit à Bernoulli «J'appelle une monade pleine et entière, c'est-à-dire une substance singulière, non pas tant une âme que l'animal lui-même ou son analogue, l'âme en tant que forme et pourvue d'un corps organique $»^{72}$ ou à Rémond «Une veritable substance (telle qu'un animal) est composée d'une ame immaterielle et d'un corps organique, et c'est le Composé et ces deux qu'on appelle Unum per se ${ }^{73}$; la substance complète, véritable, inclut le corps organique et, en cela, il n'y a de facto que des substances composées, c'est-à-dire des substances corporelles, ou encore des êtres vivants ${ }^{74}$.

Mais au sein de cette substance composée qui est une substantiellement, les substances simples restent la source de l'activité qui anime la substance composée. Dans le De Ipsa Natura, Leibniz explique : « on peut en conclure que, dans la substance corporelle, il doit se trouver une entéléchie première, une certaine capacité première d'activité [...]. Et c'est ce même principe

70. Leibniz, Remarques sur les Objections de M. Foucher, GP VI, p. 492.

71. « Il n'y a point de partie de la matiere qui ne soit divisée actuellement, et qui ne contienne des corps organiques ; [...] il y a aussi des ames par tout, comme il y a par tout des corps ; [...] les corps organiques ne sont jamais sans ames, et [...] les ames ne sont jamais separées de tout corps organique », Leibniz, Considérations sur les Principes de Vie, et sur les Natures Plastiques, par l'Auteur du Système de l'Harmonie preetablie, GP VI, p. 545.

72. «Monadem completam seu substantiam singularem voco non tam animam, quam ipsum animal aut analogum, anima vel forma et corpore organico praeditum », Leibniz à Bernoulli, Leibnizens mathematische Schriften, vol. III, Berlin, C. I. Gerhardt, 1875-1890, p. 542. Traduction par nos soins.

73. Leibniz à Rémond, GP III, p. 657.

74. Ici, «substance composée », «substance corporelle» et «être vivant» peuvent être considérés comme synonymes. Comme Leibniz l'écrit à Des Bosses : «quoniam revera nullam substantiam corpoream admittendam puto, nisi ubi est corpus organicum cum Monade dominante, seu vivum, animal scilicet, vel animali analogum » (GP II, p. 481-482) et « ita substantiam corpoream seu compositam restringo ad sola viventia » (GP II, p. 520). 
substantiel qui, dans les vivants, s'appelle âme, dans les autres êtres, forme substantielle, et qui, en tant qu'il constitue avec la matière une substance véritablement une, ou une unité par soi, est ce que j'appelle Monade. Supprimez ces unités véritables et réelles : les corps ne seront plus que des êtres par agrégation, ou plutôt, c'en est la conséquence, ne seront plus de véritables êtres ${ }^{75}$; au sein d'une substance composée, les substances simples animent l'ensemble des corps organiques et, par cette animation active, font du tout organique une unité bien fondée. En d'autres termes, l'activité et l'unité du corps organique dérivent de celles des substances simples. En ce sens, même entendues au sein d'une substance composée, dans la sorte d'imbrication monado-corporelle, les substances simples restent la source de l'activité authentique qui anime le tout de la substance composée ; de ce fait, les substances simples sont toujours le garant de la substantialité du tout.

En effet, les substances simples contiennent la loi intrinsèque qui gouverne le développement du tout. Dans la suite du De Ipsa Natura, Leibniz considère l'entéléchie première, ou puissance primitive active (c'està-dire le cœur perceptif actif, le noyau de la simplicité monadique) comme la lex insita ${ }^{76}$ de la substance : l'activité perceptive caractérisant le simple est la seule loi, le seul programme d'action ${ }^{77}$, qui gouverne, donne une direction au développement de la substance composée. Comme Leibniz l'écrit à Burnett, « chaque animal et chaque plante aussi est une substance corporelle, ayant en soy le principe de l'unité, qui fait que c'est veritablement une substance et non pas un aggregé $\gg^{78}$ : la substantialité de la substance composée vient de la substantialité des substances simples qui la constituent et, en cela, la simplicité reste le noyau ontologique, le garant de la substantialité. Aussi est-ce peut-être en ce sens que l'on peut comprendre l'ellipse, dans le premier paragraphe des Principes de la Nature et de la Grace, qui définit la substance composée comme «assemblage des substances simples, ou de Monades ${ }^{79}$ et passe sous silence, par la même occasion, l'incarnation organique que la substance composée suppose : un tel geste définitionnel peut inciter à considérer que même dans la substance composée,

75. «Hinc judicari potest, debere in corporea substantia reperiri entelechiam primam, tandem pròton dektikòn activitatis [...]. Atque hoc ipsum substantiale principium est, quod in viventibus anima, in aliis forma substantialis appellatur, et quatenus cum materia substantiam vere unam, seu unum per se constituit, id facit quod ego Monadem appello, cum sublatis his veris et realibus unitatibus, non nisi entia per aggregationem, imo quod hinc sequitur, nulla vera entia in corporibus sint superfutura », Leibniz, De Ipsa Natura, GP IV, p. 511. Traduction P. Schrecker, Leibniz, Opuscules philosophiques choisis, op. cit., p. 219.

76 Leibniz, De Ipsa Natura, GP IV, p. 512.

77. «In fact, the very composition to infinity of a natural machine suggests that it is individuated by a single law or a single program of action», O. Nachtomy, «Leibniz on Artificial and Natural Machines : On What It Means to Remain a Machine to the Least of Its Parts », op. cit., p. 75.

78. Leibniz à Burnett, GP III, p. 260.

79. Leibniz, Principes de la Nature et de la Grace, fondés en raison, GP VI, p. 598. 
l'investigation ontologique se fonde avant tout sur les substances simples et que, de ce fait, la simplicité reste le noyau substantiel fondamental.

Dans cette régression vers la simplicité, on peut aussi noter que le double modèle intégratif et élémentaire présenté en introduction se retrouve ici. [2] Dans une conception bottom-up de l'attribution substantielle, la substantialité de la substance composée peut être réduite à la substantialité des simples monadiques qui la constitue. Et, [1] dans une conception top-down de l'attribution substantielle, la substantialité de la substance composée peut être réduite au simple monadique qui anime le corps organique dans son entier, c'est-à-dire au simple de la monade dominante ${ }^{80}-$ et ici, nous retrouvons l'idée du simple comme lex insita qui dirige le développement du tout de l'être vivant. Ainsi, au sein des substances composées, toute marque substantielle pointe vers le simple et met de ce fait pleinement en avant le rôle de garant substantiel de la simplicité monadique; la définition des monades en termes de substances simples en devient ainsi pleinement justifiée.

\section{Conclusion : les monades, substances simples}

La recherche leibnizienne sur les fondements de la substantialité et sur les réquisits ontologiques s'élabore avant tout en termes d'unité, en témoigne la formule résumant l'investigation menée dans la correspondance avec Arnauld, «ce qui n'est pas véritablement un être n'est pas non plus véritablement un $\hat{e} t r e ~{ }^{81}$; cette unité véritable est définie et délimitée en la confrontant à l'unité arithmétique, à la notion de point géométrique, ou encore à l'atome physique. Face à ce développement consacré à la notion d'unité, la caractérisation substantielle par le simple semble beaucoup plus mince ; ainsi, est simple ce qui est sans parties et dont la nature est d'avoir de la perception et, par conséquent, de représenter les choses composées.

Quoique tenant en quelques mots, cette définition précise du simple est exhaustive. En ce que le simple est ce qui est sans parties, le simple est par conséquent indivisible, immatériel et impérissable. Regroupant les qualités de l'unité véritable, le simple englobe aussi son rôle de garant de substantialité. En ce que le simple est ce qui a de la perception, le simple se dote par conséquent d'un contenu perceptif foisonnant qui le présente d'emblée comme un simple riche de complexité. De cette complexité

80. «An animal, for example, is one machine of nature because the functions of the entire composite are "caused" by one central monad; this central monad contains within it the reasons for what is to happen within the composite substance. And, in a certain sense, the very fact that one central or dominant monad contains reasons for everything that happens within its subordinates is all that there is to the claim that a monad "unifies" its subordinates into one machine of nature », B. Look, "On Monadic Domination in Leibniz's Metaphysics », British Journal for the History of Philosophy, 10, (2002), p. 393.

81. Leibniz à Arnauld, GP II, p. 97. 
perceptive jaillit une activité authentique qui, caractéristique de la substantialité, manifeste de nouveau le simple comme le noyau ontologique garant de substantialité. En outre, en tant que ce qui a de la perception représente conséquemment les choses composées, de facto, les substances simples sont jointes à des corps organiques, ces derniers fournissant les moyens physiques d'une réalisation concrète de l'expression perceptive monadique. Imbriqués à l'infini, substances simples et corps organiques forment des substances composées au sein desquelles le simple apparaît toujours comme la source ontologique, le garant de la substantialité de l'ensemble formé par les substances simples et les corps organiques. En ce sens, en caractérisant le simple comme ce qui est sans parties et dont la nature est d'avoir de la perception et, par là-même, de représenter les choses composées, Leibniz rend la notion de simplicité centrale dans l'examen substantiel tant est si bien que la définition des monades comme substances simples trouve tout son sens.

Ainsi, même si Leibniz énonce une définition précise et exhaustive du simple, son appréhension n'en est pas immédiate pour autant. En effet, la qualification des monades en termes d'unités véritables ouvre une dialectique de l'un et du multiple qui semble perdurer dans la qualification des monades par le simple. Cet usage persistant de l'un et du multiple trouve peut-être son sens en ce que la substance simple est distinguée de la substance composée et que la composition peut s'analyser par l'un et le multiple. Pourtant, la conception des monades comme substances simples, nous l'avons vu, quoiqu'englobant les développements sur l'unité véritable, contient plus. En cela, cette conception ouvrirait sa propre dialectique du simple et du complexe. Cette lecture d'une simplicité riche de complexité accompagne la relecture des rapports entre substances simples et substances composées que mènent ceux, comme M. Fichant ou J.E. H. Smith, qui mettent en avant la biologisation de la métaphysique chez le dernier Leibniz $^{82}$. Plus généralement, cette simplicité riche de complexité rend plus compréhensible le contenu de la simplicité monadique chez Leibniz. En ce sens, quoique déjà soulignée par certains comme A. Becco, J.-P. Anfray ou T.-A. Hillman, cette lecture mérite qu'on s'y attarde davantage.

82. J. E. H. Smith, « The Body-Machine in Leibniz's Early Physiological and Medical Writings: A Selection of Texts with Commentary », The Leibniz Review, 17, (2007), p. 141-179. 